\title{
Deoxynivalenol and Nivalenol Production by Fusarium culmorum Isolates Differing in Aggressiveness Toward Winter Rye
}

\author{
G. Gang, T. Miedaner, U. Schuhmacher, M. Schollenberger, and H. H. Geiger
}

First and fifth authors: Institute of Plant Breeding, Seed Science, and Population Genetics (350); second author: State Plant Breeding Institute (720); third and fourth authors: Institute of Animal Nutrition (450), University of Hohenheim, D-70593 Stuttgart, Germany. Accepted for publication 20 May 1998.

\begin{abstract}
Gang, G., Miedaner, T., Schuhmacher, U., Schollenberger, M., and Geiger, H. H. 1998. Deoxynivalenol and nivalenol production by Fusarium culmorum isolates differing in aggressiveness toward winter rye. Phytopathology 88:879-884.

A susceptible synthetic winter rye population was inoculated with 42 isolates of Fusarium culmorum, originating from nine European countries and Australia, at two field locations in Germany. Significant $(P=$ 0.01 ) genetic variation in aggressiveness of isolates of $F$. culmorum was observed across both field locations. Field samples were used to determine deoxynivalenol (DON), nivalenol (NIV), and ergosterol (ERG) contents. The 42 isolates also were incubated on rye grain in vitro, and DON and NIV contents were analyzed. Thirty-four isolates produced DON, and seven isolates produced NIV at both field locations and in vitro. Mean DON contents ranged from 0.5 to $64.6 \mathrm{mg} / \mathrm{kg}$ in grain from field trials and
\end{abstract}

ABSTRACT

Head blight, caused by Fusarium culmorum (W. G. Sm.) Sacc., is one of the most destructive diseases of wheat and rye (Secale cereale L.). It causes yield reductions and contaminates grain with mycotoxins. The latter constitute a potential health risk for humans and animals. The trichothecene deoxynivalenol (DON) frequently occurs in naturally infected crops, and nivalenol (NIV) also may occur. NIV is considered more toxic than DON (33). In literature from around the world, mean DON concentrations in positive samples ranged from 0.03 to $1.78 \mathrm{mg} / \mathrm{kg}$ in wheat grain, with maximum levels up to $8.53 \mathrm{mg} / \mathrm{kg}$ (29). In southwestern Germany, levels as high as $20.5 \mathrm{mg}$ of DON per $\mathrm{kg}$ were detected in naturally infected wheat samples (19). In rye, DON concentrations of 0.01 to $1.05 \mathrm{mg} / \mathrm{kg}$ in grain have been reported (6). Recently, researchers $(1,10,17)$ found that $F$. culmorum isolates from Japan, New Zealand, and the United States produce NIV.

The phytotoxic nature of DON $(34,35)$ suggests its production may be a component of the pathogenicity (i.e., ability to cause disease) and aggressiveness (i.e., extent of disease) of $F$. culmorum isolates. Manka et al. (11) found that F. culmorum isolates that did not form DON were still pathogenic toward rye, wheat, and triticale seedlings. Proctor et al. (22) reported that a trichothecene-deficient $F$. graminearum isolate generated by gene disruption was pathogenic on wheat and rye but was less aggressive than the wild-type. These findings indicate that DON formation is not required for pathogenicity but may play a role in the aggressiveness of F. culmorum isolates. To test this hypothesis, a survey

Corresponding author: T. Miedaner

E-mail address: miedaner@uni-hohenheim.de

Publication no. P-1998-0706-02R

(C) 1998 The American Phytopathological Society from 0.3 to $376.3 \mathrm{mg} / \mathrm{kg}$ in grain incubated in vitro; mean NIV contents ranged from 17.6 to $30.4 \mathrm{mg} / \mathrm{kg}$ in grain from field trials and from 0.8 to $381.0 \mathrm{mg} / \mathrm{kg}$ in grain incubated in vitro. No correlation was found between the DON content of field-grown grain and grain incubated in vitro. NIV-producing isolates originated from the Netherlands, Germany, Italy, and Australia. More aggressive isolates produced higher mean DON contents in grain in field trials $(r=0.69 ; P=0.01)$. However, DON production rate per unit of fungal biomass, estimated as the DON/ERG ratio at harvest, was not correlated with aggressiveness. Toxin production seemed to be a common feature in $F$. culmorum. In vitro assays reliably distinguished DON- and NIV-producing types of F. culmorum; however, these assays could not predict production of DON by these isolates in the field.

Additional keywords: head blight, mycotoxins, population genetics, Secale cereale, trichothecenes. of a representative sample of isolates collected from natural sources is necessary. However, DON production by isolates, as well mycelium growth rate within the host, may vary according to the genotype of the isolate. The latter can be assessed by the ergosterol (ERG) content of grain (27). To estimate the DON production rate by isolates in relation to their aggressiveness, the DON/ ERG ratio, therefore, is more appropriate than determining only the total DON content of grain.

In the current study, we analyzed 42 isolates of $F$. culmorum that had already been tested for their aggressiveness in causing head blight in five field environments (location-year combinations [13]). All isolates were pathogenic, and their aggressiveness varied continuously. The objectives of this study were (i) to estimate genetic variation for DON and NIV production among $42 \mathrm{~F}$. culmorum isolates, (ii) to correlate DON production in field-grown and in vitro-incubated rye grain, and (iii) to compare the DON content and DON/ERG ratio to the head blight rating.

\section{MATERIALS AND METHODS}

Isolates. For this study, 42 single-spore isolates of $F$. culmorum, described in detail by Miedaner et al. (13), were used. The isolates originated from nine European countries and Australia. All isolates were identified as $F$. culmorum based on conidial morphology, according to Gerlach and Nirenberg (7), and species-specific polymerase chain reaction (PCR)-based DNA analysis (23). Isolates were stored in liquid nitrogen until used to produce inoculum.

Field trials. The design of field trials, inoculum production, plant inoculation, and disease assessment were carried out as previously described (13). Briefly, conidia for inoculation were produced in 1-liter glass flasks containing $500 \mathrm{ml}$ of boiled and sterilized wheat grain. The cultures were incubated in the dark at $13^{\circ} \mathrm{C}$ for 4 weeks. 
After incubation, grain was removed and placed in plastic boxes $\left(40 \times 60 \mathrm{~cm}^{2}\right)$ and covered with punctured polyethylene foil. Boxes were placed under permanent black light for 6 to 14 days and airdried. For inoculum preparation, conidia were washed from the wheat grain with tap water, adjusted to a concentration of $3 \times 10^{5}$ spores per $\mathrm{ml}$, and Tween 20 (final concentration of $0.01 \%$ ) was added to the suspension.

The inoculum was sprayed on a synthetic winter rye population (SYN 8392) grown in two-row microplots $\left(0.42 \mathrm{~m}^{2}\right)$ at two locations (Stuttgart-Hohenheim, Germany, at $400 \mathrm{~m}$ above sea level, $8.5^{\circ} \mathrm{C}$ mean annual temperature, and $685-\mathrm{mm}$ mean annual precipitation and Bad Schönborn near Heidelberg, Germany, at $218 \mathrm{~m}$ above sea level, $10.2^{\circ} \mathrm{C}$ mean annual temperature, and $730-\mathrm{mm}$ mean annual precipitation) in 1992. Isolates were randomized in a seven by six lattice design with four replications. Ten noninoculated microplots were located adjacent to each replication as control plots. Each treated plot was inoculated once with $60 \mathrm{ml}$ of spore suspension at the mid-flowering stage. The plots were separated by four rows of noninoculated winter rye $\left(0.83 \mathrm{~m}^{2}\right)$ to minimize plot-by-plot interference.

TABLE 1. Means of head blight rating, deoxynivalenol (DON) and ergosterol (ERG) contents, and DON/ERG ratio in grain harvested in the field (two locations in Germany) and incubated in vitro after inoculation of a synthetic winter rye population with 42 Fusarium culmorum isolates

\begin{tabular}{|c|c|c|c|c|c|}
\hline \multirow[b]{2}{*}{$\begin{array}{l}\text { F. culmorum } \\
\text { code }\end{array}$} & \multicolumn{4}{|c|}{ Field } & \multirow{2}{*}{ 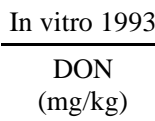 } \\
\hline & $\begin{array}{l}\text { Head blight } \\
\text { rating }^{\mathrm{a}}\end{array}$ & $\begin{array}{c}\mathrm{DON} \\
(\mathrm{mg} / \mathrm{kg})\end{array}$ & $\begin{array}{c}\text { ERG } \\
(\mathrm{mg} / \mathrm{kg})\end{array}$ & $\begin{array}{l}\text { DON/ERG } \\
\text { ratio }\end{array}$ & \\
\hline 01 & 2.90 & 1.10 & 30.62 & 0.04 & 0.4 \\
\hline 02 & 3.45 & 23.30 & 40.52 & 0.58 & 0.9 \\
\hline 03 & 4.07 & 0.70 & 42.05 & 0.02 & 2.3 \\
\hline 04 & 4.12 & 29.25 & 30.26 & 0.97 & 16.7 \\
\hline 05 & 4.05 & 1.60 & 31.86 & 0.05 & 0.5 \\
\hline 06 & 4.40 & 0.90 & 47.18 & 0.02 & 0.5 \\
\hline 07 & 4.54 & 5.95 & 45.43 & 0.13 & 0.3 \\
\hline 08 & 4.56 & 5.85 & 44.93 & 0.13 & 1.4 \\
\hline 09 & 4.61 & 1.45 & 31.73 & 0.05 & 1.7 \\
\hline 10 & 3.95 & 1.40 & 40.62 & 0.03 & 0.6 \\
\hline 11 & 4.99 & 20.30 & 64.08 & 0.32 & 63.7 \\
\hline 12 & 4.23 & 28.35 & 36.53 & 0.78 & 195.8 \\
\hline 13 & 4.84 & 41.75 & 79.21 & 0.53 & 131.8 \\
\hline 14 & 5.37 & 26.15 & 52.72 & 0.50 & 207.4 \\
\hline 15 & 5.74 & 39.10 & 75.50 & 0.52 & 120.1 \\
\hline 16 & 4.94 & 40.80 & 56.78 & 0.72 & 211.9 \\
\hline 17 & 4.85 & 26.45 & 61.29 & 0.43 & 94.1 \\
\hline 18 & 5.30 & 34.75 & 49.00 & 0.71 & 376.3 \\
\hline 19 & 5.44 & 41.60 & 57.99 & 0.71 & 70.9 \\
\hline 20 & 5.74 & 27.25 & 42.08 & 0.65 & 173.1 \\
\hline 21 & 5.43 & 42.50 & 50.64 & 0.84 & 207.2 \\
\hline 22 & 5.84 & 29.40 & 50.09 & 0.59 & 112.0 \\
\hline 23 & 5.44 & 42.00 & 63.98 & 0.66 & 3.1 \\
\hline 24 & 5.82 & 49.30 & 66.21 & 0.75 & 221.1 \\
\hline 25 & 6.10 & 47.40 & 53.61 & 0.88 & 235.5 \\
\hline 26 & 6.66 & 36.85 & 68.96 & 0.54 & 251.2 \\
\hline 27 & 5.45 & 41.85 & 53.74 & 0.80 & 150.1 \\
\hline 28 & 6.21 & 54.20 & 72.14 & 0.75 & 106.4 \\
\hline 29 & 6.48 & 60.25 & 80.68 & 0.75 & 204.6 \\
\hline 30 & 6.35 & 44.00 & 60.88 & 0.72 & 222.5 \\
\hline 31 & 6.66 & 44.95 & 66.97 & 0.67 & 206.3 \\
\hline 32 & 6.53 & 45.65 & 69.11 & 0.66 & 238.3 \\
\hline 33 & 6.65 & 49.45 & 74.63 & 0.67 & 73.2 \\
\hline 34 & 6.59 & 41.95 & 59.57 & 0.71 & 266.3 \\
\hline 35 & 6.42 & 45.75 & 85.56 & 0.54 & 98.9 \\
\hline 36 & 6.50 & 37.00 & 65.02 & 0.57 & 54.6 \\
\hline 37 & 6.65 & 51.00 & 78.60 & 0.65 & 215.5 \\
\hline 38 & 6.68 & 44.70 & 78.88 & 0.57 & 104.2 \\
\hline 39 & 6.50 & 43.30 & 46.53 & 0.94 & 199.5 \\
\hline 40 & 7.07 & 58.10 & 92.16 & 0.64 & 7.5 \\
\hline 41 & 6.58 & 40.20 & 65.75 & 0.62 & 348.1 \\
\hline 42 & 7.05 & 50.30 & 61.85 & 0.81 & 15.1 \\
\hline Mean & 5.50 & 33.36 & 57.76 & 0.56 & 124.6 \\
\hline
\end{tabular}

a Based on a 1 (no symptoms visible) to 9 (96 to $100 \%$ of all spikelets per plot diseased) scale.
Head blight severity was assessed several times, based on a scale of 1 (no symptoms visible) to 9 (96 to $100 \%$ of all spikelets in the plot diseased [13]), beginning at the onset of symptom development and continuing until the beginning of yellow ripening. At Hohenheim, ratings were carried out 24, 27, 30, 32, and 34 days after inoculation and at Bad Schönborn 19, 23, 26, and 27 days after inoculation. Arithmetic means of the head blight ratings across all assessment dates were used for further analyses. At the yellowripening stage, 60 arbitrarily chosen heads per plot were harvested by hand, carefully threshed in a single-head thresher, and the grain was ground in a mill (Cyclotec sample mill, Tecator, Höganäs, Sweden) with a $0.8-\mathrm{mm}$ mesh sieve. For toxin determination, the flour from all four replications from each location was mixed and analyzed as one sample due to a shortage in lab facilities. Statistical analyses were performed on an entry mean basis, and genotypes and locations were used as sources of variance.

In vitro experiments. The same $42 F$. culmorum isolates used in the field experiments were incubated on rye grain in vitro in 1993, and the NIV-producing isolates were incubated again in 1996. For this purpose, $20 \mathrm{~g}$ of rye grain was adjusted to a moisture content of $40 \%$ with water and autoclaved in glass jars $(6 \mathrm{~cm}$ diameter, $8 \mathrm{~cm}$ height) closed with Magenta cups (Magenta Corp., Chicago). The grain was mixed with $2 \mathrm{ml}$ of water suspension containing $2.5 \times 10^{5}$ spores per $\mathrm{ml}$ under sterile conditions and incubated at $28^{\circ} \mathrm{C}$ in the dark for 2 weeks. For each isolate, two jars of grain were produced. After harvesting, grain was stored at $-20^{\circ} \mathrm{C}$ until toxin extraction.

Toxin extraction and analysis. Extraction of toxins from grain was carried out according to Tanaka et al. (32). In brief, toxins were extracted from $20 \mathrm{~g}$ of flour (field experiment) or ground, colonized kernels (in vitro experiment) with $200 \mathrm{ml}$ of acetonitrile/water (3:1, vol/vol). For final cleaning, extracts were placed in a chromatographic column $\left(30 \times 2.2 \mathrm{~cm}^{2}\right)$ with $20 \mathrm{~g}$ of florisil. Analysis of DON was performed according to Scott et al. (26). Briefly, after derivatization with Tri-Sil TBT (Pierce Chemical Co., Rockford, IL), DON was analyzed by gas-chromatography electron-capture detection (GC-ECD) and showed a peak at the expected retention time of $31.17 \mathrm{~min}$ for most isolates. In the field and in vitro experiments, however, samples of seven isolates had a peak on the GC-ECD chromatogram at a retention time of $\approx 40.86 \mathrm{~min}$. At this retention time, NIV is expected. This retention time was confirmed with a NIV standard from Wako Pure Chemical Industries Ltd., Osaka, Japan. To confirm that the isolates produced NIV, one sample from the field-grown plots of the respective isolates was analyzed additionally by gas-chromatography mass-spectrometry (GC-MS). Derivatization of extracts by trifluoroacetylation was done according to Kientz and Verweij (9). Conditions for GC-MS are described in detail by Schwadorf and Müller (25). NIV was determined according to retention time and mass spectrum and quantified with mass 583 .

Because these F. culmorum isolates were the first European isolates recognized as NIV producers, we incubated the isolates a

TABLE 2. Head blight rating, deoxynivalenol (DON) and ergosterol (ERG) contents, and DON/ERG ratio means at two field locations in Germany; genetic coefficient of variation $(\mathrm{CV}, \%)$; and heritability $\left(h^{2}\right)$ after inoculation of a synthetic winter rye population with 42 Fusarium culmorum isolates

\begin{tabular}{lcccc}
\hline & \multicolumn{2}{c}{ Mean } & & \\
\cline { 2 - 4 } Trait & Hohenheim & Bad & & \\
Schönborn & CV $(\%)^{\mathrm{a}}$ & $h^{2}$ \\
\hline Head blight rating $\mathrm{b}$ & 6.0 & 5.0 & 18.2 & 0.87 \\
DON content (mg/kg) & 35.0 & 31.7 & 49.3 & 0.89 \\
ERG content (mg/kg) & 55.1 & 60.4 & 22.0 & 0.63 \\
DON/ERG ratio & 0.63 & 0.50 & 44.7 & 0.82 \\
\hline
\end{tabular}

a Significant genotypic differentiation among isolates at $P=0.01$ ( $F$ test of corresponding mean squares) for all traits.

b Based on a 1 (no symptoms visible) to 9 (96 to $100 \%$ of all spikelets per plot diseased) scale. 
second time in vitro on rye grain in 1996, using the method described above, and determined the DON and NIV contents by GCMS following the same extraction procedure. Final cleaning, however, was performed by solid-phase chromatography with a florisil column followed by cleanup with a cation-exchange resin. Derivatization of extracts and GC-MS conditions followed methods described previously (Kientz and Verweij [9] and Schwadorf and Müller [25], respectively). Trichothecenes were measured with chemical ionization and isobutane as reagent gas.

ERG contents were determined in grain from the field trials according to the methods described by Schwadorf and Müller (24). In brief, $5 \mathrm{~g}$ of flour was saponificated with $75 \mathrm{ml}$ of $\mathrm{KOH}$ dissolved in methanol $(750 \mathrm{ml}$ of analytical grade methanol plus $100 \mathrm{~g}$ of $\mathrm{KOH}$ pellets) and extracted two times with $40 \mathrm{ml}$ of $n$ hexane each. Analysis was done by high-pressure liquid chromatography.

Statistical analyses. All analyses were calculated using the data for each location. The mean head blight rating for the noninoculated treatment did not exceed 1.4 across both locations, indicating virtually no visible infection. The values of the inoculated treatment for this trait, therefore, were used directly for analyses of variance (ANOVA) in the four replicates. For head blight rating, ANOVAs were performed on the data from each location. Entry means from individual experiments followed a normal distribution, and error variances were homogeneous according to Bartlett's test (28). Adjusted entry means and effective error mean squares (MS) were used to compute the combined analysis across both locations according to Cochran and Cox (2), with the restriction that the $F$ tests were approximate. For each missing value, $1 \mathrm{df}$ was subtracted from the error variance. Estimates of variance components for $\sigma_{g}^{2}$ (genotypic variance), $\sigma_{g l}^{2}$ (genotype-location interaction variance), and $\sigma_{e}^{2}$ (error variance) were calculated as described by Snedecor and Cochran (28) for factorial designs. Broadsense heritabilities $\left(h^{2}\right)$ on an entry-mean basis were estimated by partitioning phenotypic variance according to the formula presented by Fehr (5): $h^{2}=\sigma_{g}^{2} /\left(\sigma_{e}^{2} / r l+\sigma^{2}{ }_{g l} / l+\sigma_{g}^{2}\right)$, where $r=$ number of replicates and $l=$ number of locations.

In the ANOVAs for DON and ERG contents and DON/ERG ratio, locations were treated as replicates, resulting in estimates of variance components for $\sigma_{g}^{2}$ and $\sigma_{g l}^{2}$. The latter included the expected value of the MS of $\sigma_{e}^{2}$ and, therefore, was inflated and not given in the paper. Instead of this parameter, the correlation between both locations was used to estimate the amount of genotype-location interaction. The genotypic variance was estimated correctly by this procedure and was used for further calculation of the genotypic coefficient of variation (percentage of $\mathrm{CV}$ ) accord- ing to the formula $\left(\sigma_{g} /\right.$ mean $) \times 100$. This standardized parameter allowed comparison of all traits assessed. For the estimation of heritability of DON and ERG contents and DON/ERG ratio, the formula given above was adjusted as follows: $h^{2}=\sigma^{2}{ }_{g} /\left(M S_{g l} / l+\right.$ $\sigma_{g}^{2}$ ) (28). Phenotypic correlations between traits and locations were estimated on an entry-mean or plot basis, respectively, by standard procedures (18). All computations were calculated with the computer program package PLABSTAT (H. F. Utz, University of Hohenheim, Institute of Plant Breeding, Seed Science, and Population Genetics, Stuttgart, Germany). The effects of genotypes, locations, and replicates were assumed to be random variables.

\section{RESULTS}

All F. culmorum isolates caused visible head blight symptoms in field experiments. Head blight ratings ranged from 2.9 to 7.1 , averaging 5.5 across both locations (Table 1). Accordingly, the DON and ERG contents and DON/ERG ratio showed high variation among isolates. The DON concentration produced in vitro was, on average, approximately four times higher than DON accumulation in field-grown rye grain, ranging from 0.3 to $376.3 \mathrm{mg} / \mathrm{kg}$ of grain. The amount of DON produced in field-grown grain and grain incubated in vitro was not significantly correlated $(r=0.25: P>$ 0.1 ) when calculated with isolates producing DON in significant amounts (F. culmorum [FC] code: FC 02, FC 04, and FC 11 to 42). Head blight rating, DON and ERG contents, and DON/ERG ratio means indicated similar disease severity and mycotoxin production level in both locations (Table 2). Genotypic CV was approximately twofold higher for DON content and DON/ERG ratio than for ERG content. Genotypic differentiation among isolates was highly significant $(P=0.01)$ throughout the trials, as revealed by the $F$ test of the genotypic MS in the ANOVA. Coefficients of correlation between both locations were significant in all instances $(P=0.01)$ and were considerably higher for head blight rating, DON content, and DON/ERG ratio $(r=0.78,0.79$, and 0.72 , respectively) than for ERG content ( $r=$ 0.48 ). Consequently, heritability estimates were high for head blight rating, DON content, and DON/ERG ratio but moderate for ERG content.

Toxin analyses identified two chemotypes of F. culmorum. The first chemotype (34 isolates) produced high concentrations of DON (20.3 to $60.3 \mathrm{mg} / \mathrm{kg}$; Table 1). A second group of seven isolates produced high concentrations of NIV under field conditions (Table 3). DON concentrations in these samples were negligible compared to the average DON content of grain colonized by isolates of the DON chemotype. One isolate (FC 01) did not produce DON or

TABLE 3. Geographic origin, source, deoxynivalenol (DON) and nivalenol (NIV) contents in grain harvested in the field (mean of two locations in Germany) and incubated in vitro in two different years after inoculation with 7 NIV- and 34 DON-producing Fusarium culmorum isolates

\begin{tabular}{|c|c|c|c|c|c|c|c|c|c|}
\hline \multirow[b]{2}{*}{$\begin{array}{l}\text { F. culmorum } \\
\text { code }\end{array}$} & \multirow[b]{2}{*}{ Country } & \multirow[b]{2}{*}{ Supplier } & \multirow[b]{2}{*}{$\begin{array}{l}\text { Original } \\
\text { code }\end{array}$} & \multicolumn{2}{|c|}{ Field } & \multicolumn{2}{|c|}{ In vitro 1993} & \multicolumn{2}{|c|}{ In vitro 1996} \\
\hline & & & & $\begin{array}{l}\mathrm{DON}^{\mathrm{a}} \\
(\mathrm{mg} / \mathrm{kg})\end{array}$ & $\begin{array}{l}\mathrm{NIV}^{\mathrm{b}} \\
(\mathrm{mg} / \mathrm{kg})\end{array}$ & $\begin{array}{l}\mathrm{DON}^{\mathrm{a}} \\
(\mathrm{mg} / \mathrm{kg})\end{array}$ & $\mathrm{NIV}^{\mathrm{a}, \mathrm{c}}$ & $\begin{array}{l}\mathrm{DON}^{\mathrm{b}} \\
(\mathrm{mg} / \mathrm{kg})\end{array}$ & $\begin{array}{l}\mathrm{NIV}^{\mathrm{b}} \\
(\mathrm{mg} / \mathrm{kg})\end{array}$ \\
\hline \multicolumn{10}{|c|}{ NIV-producing isolates } \\
\hline 05 & Germany & H. Schmitz-Elsherif & $289^{\mathrm{d}}$ & 1.6 & 23.0 & 0.5 & + & 0.5 & 207 \\
\hline 06 & Australia & L. W. Burgess & F 4007 & 0.9 & 30.4 & 0.5 & + & 0.2 & 15.6 \\
\hline 07 & Italy & A. Logrieco & ATCC $56089^{d}$ & 6.0 & 25.6 & 0.3 & + & 0 & 0.8 \\
\hline 10 & Netherlands & C. H. A. Snijders & SVP $8901^{\mathrm{d}}$ & 1.4 & 27.2 & 0.6 & + & 0.6 & 381 \\
\hline \multicolumn{4}{|c|}{$\begin{array}{l}\text { DON-producing isolates } \\
\quad(\text { mean, } N=34)\end{array}$} & 40.6 & 0 & 153.1 & - & $\mathrm{ND}^{\mathrm{e}}$ & ND \\
\hline \multicolumn{4}{|c|}{ Noninoculated } & 0.5 & 0 & ND & ND & 0.03 & 0 \\
\hline
\end{tabular}

a Analyzed by gas-chromatography electron-capture detection (GC-ECD).

${ }^{\mathrm{b}}$ Analyzed by gas-chromatography mass-spectrometry (GC-MS).

${ }^{c}+$ indicates a peak on the GC-ECD chromatogram at the NIV retention time (40.86 min); - indicates no peak on the GC-ECD chromatogram at the NIV retention time.

d Additionally identified as F. culmorum by H. Nirenberg, Biologische Bundesanstalt für Land- und Forstwirtschaft, Berlin.

e Not determined. 
NIV in large concentrations. In noninoculated grain, no NIV and low concentrations of DON $(0.5 \mathrm{mg} / \mathrm{kg})$ were detected. The mean head blight ratings of field plots inoculated with NIV-producing isolates were somewhat lower than that of DON-producing isolates (4.3 versus 5.8). Patterns of GC-ECD chromatograms of in vitro samples were identical to those of the field experiments in 1993, confirming the grouping of isolates and their NIV formation on a qualitative basis. Analyzed quantitatively by GC-MS, all seven isolates again showed NIV production, with concentrations ranging from 0.8 to $381.0 \mathrm{mg} / \mathrm{kg}$ in 1996 (Table 3). Only negligible DON concentrations were found in in vitro samples colonized by NIV-producing isolates.

Correlations between head blight rating and DON content and DON/ERG ratio, respectively, were calculated only with the first group of DON-producing isolates (Fig. 1). Head blight rating and DON content of grain were significantly correlated in the field experiment (Fig. 1A). However, no association existed between head blight rating and DON/ERG ratio (Fig. 1B).

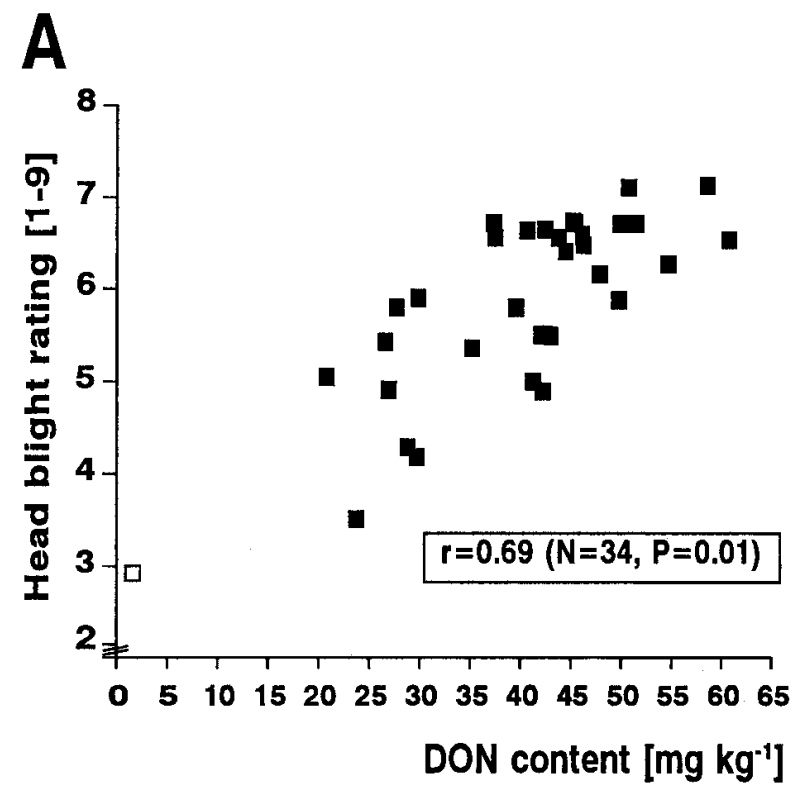

B

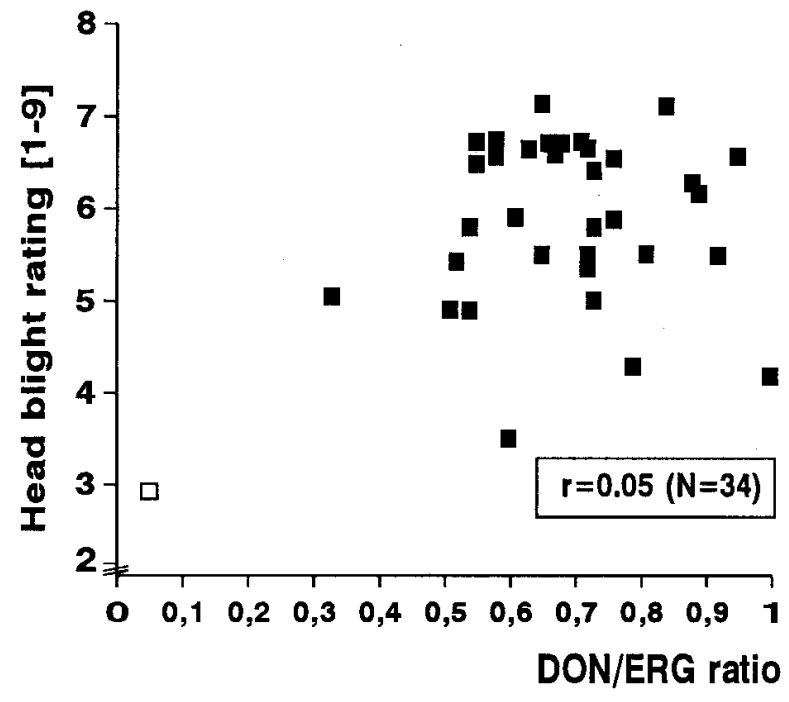

Fig. 1. Correlation between A, head blight rating (HBR, based on a scale of 1 to 9) and deoxynivalenol (DON) content and B, HBR and deoxynivalenol/ ergosterol (DON/ERG) ratio after inoculation of a synthetic winter rye population with 34 DON-producing isolates of Fusarium culmorum averaged across two field locations in Germany (ם). One isolate did not produce either DON or nivalenol in significant amounts $(\square)$.

\section{DISCUSSION}

The 42 isolates of $F$. culmorum tested showed broad genetic variation for aggressiveness, as measured by head blight rating, DON and ERG contents, and DON/ERG ratio. All traits, with the exception of ERG content, revealed high heritability estimates and highly significant genotypic CV across locations (Table 2), indicating that the environmental influence of different locations within 1 year is of minor importance for aggressiveness and mycotoxin production of $F$. culmorum isolates on a given susceptible host genotype. Because one replicate was analyzed for toxin and ERG contents, total variance can be partitioned only into variance of genotype $\left(\sigma_{g}^{2}\right)$ and genotype-location interaction variance plus error variance $\left(\sigma_{g l}^{2}+\sigma_{e}^{2}\right)$. Genotypic variance, however, is an unbiased estimate and can be used for data interpretation (28). The highly significant coefficients of correlation between locations $(r \approx 0.8)$ also support the conclusion that the majority of variation for DON and NIV contents is caused by genetic effects. High correlations were found previously for measures of aggressiveness, such as head blight rating and relative grain weight across five environments with the same set of isolates (13). The amount of fungal biomass measured as ERG obviously was influenced more by genotype-location interaction and error variance. ERG is a useful trait for estimating Fusarium biomass, because grain from artificial $F$. culmorum head blight infections contain hardly any other fungus, as revealed by isolations on agar.

Of the 42 isolates examined, 34 isolates produced DON in high concentrations, 7 isolates formed high concentrations of NIV, and 1 isolate produced none of these toxins in significant concentrations. The nontoxin producer had been cultured since 1952 and was not only weakly aggressive in the field experiment but also grew and sporulated slowly on artificial media at $18^{\circ} \mathrm{C}$. Therefore, the observed deficiency in toxin production might be due to reduced general vitality. Because most isolates were not known to produce mycotoxins at the beginning of this study, they represent an unselected sample for this trait. The data, therefore, indicate that mycotoxin production is a common feature in F. culmorum. This agrees with data on $F$. graminearum. Of $114 F$. graminearum isolates collected from soils or cereal plants on a worldwide basis, $95 \%$ were capable of producing DON in vitro (15).

Seven of the tested isolates (17\%) produced NIV; five of these originated from European countries. Although NIV-producing $F$. culmorum isolates have been identified previously in Japan (1), New Zealand (10), and the United States (17), this is, to our knowledge, the first report on European isolates forming NIV. Identification of NIV-producing isolates as F. culmorum was confirmed by taxonomists (H. Nirenberg, Berlin, for five isolates, and L. W. Burgess, Sydney, Australia, for two isolates) and species-specific PCR assays (23). The five European NIV-producing isolates were from three countries (Germany, the Netherlands, and Italy), indicating that NIV production in F. culmorum is geographically widespread in Europe. Interestingly, we found only low concentrations of DON in all samples colonized by NIV-producing isolates (Table 3). Because even samples from noninoculated plots contained some DON $(0.5 \mathrm{mg} / \mathrm{kg}$ of grain on average), this might have been caused by a small amount of natural infection with other isolates in the field. The same might be true for grain used in in vitro experiments that also were produced in the field. Generally, our F. culmorum isolates seemed to produce either DON or NIV in significant concentrations but not both toxins together. This has been reported previously for $F$. graminearum by Ichinoe et al. (8), in which isolates were classified as DON or NIV types. More recent publications, however, have reported that $F$. graminearum isolates can be found that produce both toxins in significant concentrations $(21,31)$.

The amount of DON or NIV produced across both field locations on one rye genotype was not associated with the mycotoxin contents produced in vitro on grain of the same genotype. This 
finding corroborates that environmental conditions greatly influence the quantity of mycotoxin produced. In the literature, it is well established that substrate and cultural conditions (e.g., temperature, water content, and duration of growth) greatly influence the amount of DON produced in vitro $(12,20)$. The physiology of the host in the field and pathogenesis itself may further influence toxin content, whereas autoclaved rye grain incubated in vitro with $F$. culmorum isolates may serve only as a carbon and nitrogen source without specific effects. However, all NIV- and DONproducing isolates formed the respective toxin under field and in vitro conditions. Therefore, in vitro studies can serve to distinguish the type of mycotoxin produced by isolates of F. culmorum.

Head blight rating and DON content of kernels were correlated in our study (Fig. 1A). Therefore, highly diseased rye heads can be expected to contain moderate to high amounts of DON in kernels. Similarly, in field experiments involving different winter wheat genotypes infected with $F$. culmorum or $F$. graminearum, DON content of kernels was correlated with head blight incidence (17), head blight rating, yield reduction (30), and percentage of tombstone kernels (36). However, we found considerable variation in the amount of mycelium produced by individual isolates within infected host tissue, as reflected by the ERG content, which varied from 30.3 to $92.2 \mathrm{mg} / \mathrm{kg}$ of grain (Table 1). Because isolates with higher ERG contents also tended to have higher DON contents $(r=0.69 ; P=0.01)$, the differences in DON content may reflect only the different amount of fungal biomass present in the host.

To compare the capacity for DON production among isolates, we calculated DON content relative to the amount of mycelium measured by ERG. No association existed between aggressiveness and DON/ERG ratio (Fig. 1B). The large difference in aggressiveness of isolates, therefore, cannot be explained by different DON production rates (i.e., DON content per unit of mycelium) alone. The ability of a $F$. culmorum isolate to produce DON may support the infection, but the quantity of DON produced seems to be unimportant. This finding agrees with the results of Proctor et al. (22) and Desjardins et al. (4). Their trichothecene-deficient $F$. graminearum isolate had reduced aggressiveness in greenhouse and field experiments compared to the wild-type. However, some level of aggressiveness was retained, although no DON could be found in the host. Because both studies involved only a single isolate that was totally unable to produce trichothecenes, the researchers could not compare multiple levels of DON production with different levels of aggressiveness.

Our findings with 42 isolates illustrate that although DON may play some role in expression of disease symptoms additional mechanisms must account for the actual level of aggressiveness of an isolate. However, it is impossible in field experiments with a wide array of isolates to separate cause and effect. Because DON production in vitro was not correlated with disease symptoms or in vivo production, this measure could not be used to solve this question. The use of the DON/ERG ratio, however, at least allows us to separate the effects of DON from those of fungal biomass.

Whether an earlier sample collection could provide further insights into how significantly DON influences aggressiveness needs to be investigated further. Recently, Mirocha et al. (16) showed, using single-spikelet analysis, that DON is detectable as soon as $48 \mathrm{~h}$ after inoculation. The amount of DON in this very early phase of pathogenesis may contribute more to the aggressiveness of an isolate than the amount of DON measured at harvest. Such studies should be repeated with a greater number of isolates with different levels of aggressiveness to obtain conclusive results concerning the role of DON and other mycotoxins in pathogenesis. One alternative would be to examine the aggressiveness of a greater number of isogenic strains that are disrupted in their trichothecene-producing gene(s).

Other possible causes of aggressiveness include cell-wall degrading enzymes. F. culmorum isolates grown on cell walls of wheat seedlings produce xylanase in the very early stages of in- fection (3). Therefore, we measured xylanase production by the 42 isolates after inoculation of the basal shoots of young winter rye in a greenhouse (14). In contrast to our findings for DONproduction rate, a significant correlation between the xylanase/mycelium protein ratio and aggressiveness was found $(r=0.66 ; P=$ 0.01). Thus, xylanase seems to be important for the aggressiveness of an isolate inoculated at the basal shoots of young rye plants. Further research is required to substantiate the role of lytic enzymes as putative aggressiveness factors.

Regarding the high percentage of mycotoxin producers among the 42 tested $F$. culmorum isolates, most natural head blight epidemics should result in mycotoxin accumulation in grain. NIVproducing isolates have been found less frequently than DONproducing isolates but do occur in different European countries. NIV-producing isolates showed lower aggressiveness, suggesting that NIV may be less phytotoxic than DON. Also, samples infected with NIV-producing isolates were less heavily colonized and possessed lower mean ERG contents than those of DON-producing isolates (40.5 versus $61.2 \mathrm{mg}$ of ERG per $\mathrm{kg}$ of grain). Because NIV is considerably more toxic than DON (33), however, the average toxicity of the NIV samples in our study should be higher than that of the DON-contaminated samples. To estimate the potential health hazard of $F$. culmorum-contaminated grain for animal and human consumption, therefore, it is insufficient to analyze feed and food only for DON.

\section{ACKNOWLEDGMENTS}

Research was funded by the Deutsche Forschungsgemeinschaft (DFG, Grant Ge 340/17-1), Bonn. We thank J. Freesemann for enthusiastic and skillful assistance and H.-M. Müller, W. Drochner, U. Lauber, H. TerryJara (Institute for Animal Nutrition, University of Hohenheim, Germany) and K. Schwadorf (State Institute for Agricultural Chemistry, University of Hohenheim, Germany). We also thank A. G. Schilling (Institute of Plant Breeding, Seed Science, and Population Genetics) for conducting PCR assays, H. Nirenberg (Biologische Bundesanstalt) for classifying five Fusarium culmorum isolates, and H. Wortmann (Hybro GbR) for supplying experimental ground and technical assistance. C. H. A. Snijders, (Wageningen, the Netherlands), A. Logrieco, (Bari, Italy), L. W. Burgess, (Sidney, Australia), A. Mesterházy, (Szeged, Hungary), H. Nirenberg (Berlin), H. Schmitz-Elsherif (Stuttgart, Germany) and J. Unger (Braunschweig, Germany) contributed $F$. culmorum isolates for this study.

\section{LITERATURE CITED}

1. Atanassov, Z., Nakamura, C., Mori, N., Kaneda, C., Kato, H., Jin, Y.-Z., Yoshizawa, T., and Murai, K. 1994. Mycotoxin production and pathogenicity of Fusarium species and wheat resistance to Fusarium head blight. Can. J. Bot. 72:161-167.

2. Cochran, W. G., and Cox, G. M. 1957. Experimental Designs. 2nd ed. John Wiley \& Sons, New York.

3. Cooper, R. M., Longman, D., Campbell, A., Henry, M., and Lees, P. E. 1988. Enzymatic adaptation of cereal pathogens to the monocotyledonous primary wall. Physiol. Mol. Plant Pathol. 32:33-47.

4. Desjardins, A. E., Proctor, R. H., Bai, G., McCormick, S. P., Shaner, G., Buechley, G., and Hohn, T. M. 1996. Reduced virulence of trichothecene-nonproducing mutants of Gibberella zeae in wheat field tests. Mol. Plant-Microbe Interact. 9:775-781.

5. Fehr, W. R. 1987. Principles of Cultivar Development, vol. 1: Theory and Technique. Macmillan Publishing Co., New York.

6. Gareis, M., Bauer, J., Enders, C., and Gedek, B. 1989. Contamination of cereals and feed with Fusarium mycotoxins in European countries. Pages 441-472 in: Fusarium: Mycotoxins, Taxonomy and Pathogenicity. J. Chelkowski, ed. Elsevier Science Publishing Co., Amsterdam.

7. Gerlach, W., and Nirenberg, H. I. 1982. The Genus Fusarium-A Pictorial Atlas. Mitteilungen Biologische Bundesanstalt für Land- und Forstwirtschaft, Berlin-Dahlem 209. P. Parey, Berlin.

8. Ichinoe, M., Kurata, H., Sugiura, Y., and Ueno, Y. 1983. Chemotaxonomy of Gibberella zeae with special reference to production of trichothecenes and zearalenone. Appl. Environ. Microbiol. 46:1364-1369.

9. Kientz, C. E., and Verweij, A. 1986. Trimethylsilylation and trifluoracetylation of a number of trichothecenes followed by gas chromatographic analysis on fused-silica capillary columns. J. Chromatogr. 355: 229-240. 
10. Lauren, D. R., Sayer, S. T., and diMenna, M. E. 1992. Trichothecene production by Fusarium species isolated from grain and pasture throughout New Zealand. Mycopathologica 120:167-176.

11. Manka, M., Visconti, A., Chelkowski, J., and Bottalico, A. 1985. Pathogenicity of Fusarium isolates from wheat, rye, and triticale towards seedlings and their ability to produce trichothecenes and zearalenone. Phytopathol. Z. 113:24-29.

12. Megalla, S. E., Bennet, G. A., and Shotwell, O. L. 1987. Production of deoxynivalenol and zearalenone by isolates of Fusarium graminearum Schw. J. Food Prot. 50:826-828.

13. Miedaner, T., Gang, G., and Geiger, H. H. 1996. Quantitative-genetic basis of aggressiveness of 42 isolates of Fusarium culmorum for winter rye head blight. Plant Dis. 80:500-504.

14. Miedaner, T., Gang, G., Schilling, A. G., and Geiger, H. H. 1997. Aggressiveness and mycotoxin production of populations of Fusarium culmorum and Fusarium graminearum in winter rye. Cereal Res. Commun. 25:471-475.

15. Mirocha, C. J., Abbas, H. K., Windels, C. E., and Xie, W. 1989. Variation in deoxynivalenol, 15-acetyldeoxynivalenol, 3-acetyldeoxynivalenol, and zearalenone production by Fusarium graminearum isolates. Appl. Environ. Microbiol. 55:1315-1316.

16. Mirocha, C. J., Hui, Y., Evans, C. K., Kolaczkowski, E. K., and Dill-Macky, R. 1997. Chemistry and physiology of deoxynivalenol in pathogenesis. Cereal Res. Commun. 25:309-313.

17. Mirocha, C. J., Xie, W., Xu, Y., Wilcoxson, R. D., Woodward, R. P., Etebarian, R. H., and Behele, G. 1994. Production of trichothecene mycotoxins by Fusarium graminearum and Fusarium culmorum on barley and wheat. Mycopathologia 128:19-23.

18. Mode, C. J., and Robinson, H. F. 1959. Pleiotropism and the genetic variance and covariance. Biometrics 15:518-537.

19. Müller, H.-M., and Schwadorf, K. 1993. A survey of the natural occurrence of Fusarium toxins in wheat grown in a southwestern area of Germany. Mycopathologia 121:115-121.

20. O'Neill, K., Damaglou, A. P., and Patterson, M. F. 1993. Toxin production by Fusarium culmorum IMI 309344 and F. graminearum NRRL 5883 on grain substrates. J. Appl. Bacteriol. 74:625-628.

21. Perkowski, J., Kiecana, I., Schuhmacher, U., Müller, H.-M., Chelkowski, J., and Golinski, P. 1997. Head infection and accumulation of Fusarium toxins in kernels of 12 barley genotypes inoculated with Fusarium graminearum isolates of two chemotypes. Eur. J. Plant Pathol. 103:85-90.

22. Proctor, R. H., Hohn, T. M., and McCormick, S. P. 1995. Reduced virulence of Gibberella zeae caused by disruption of a trichothecene toxin biosynthetic gene. Mol. Plant-Microbe Interact. 8:593-601.
23. Schilling, A. G., Möller, E. M., and Geiger, H. H. 1996. Polymerase chain reaction-based assays for species-specific detection of Fusarium culmorum, F. graminearum, and F. avenaceum. Phytopathology 86:515-522.

24. Schwadorf, K., and Müller, H.-M. 1989. Determination of ergosterol in cereals, mixed feed components, and mixed feeds by liquid chromatography. J. Assoc. Off. Anal. Chem. 72:457-462.

25. Schwadorf, K., and Müller, H.-M. 1991. Determination of trichothecenes in cereals by gas chromatography with ion trap detection. Chromatographia 32:137-142.

26. Scott, P. M., Kanhere, S. R., and Tarter, E. J. 1986. Determination of nivalenol and deoxynivalenol in cereals by electron-capture gas chromatography. J. Assoc. Off. Anal. Chem. 69:889-893.

27. Seitz, L. M., Sauer, D. B., Burroughs, R., Mohr, H. E., and Hubbard, J. D. 1979. Ergosterol as a measure of fungal growth. Phytopathology 69:1202-1203.

28. Snedecor, G. W., and Cochran, W. G. 1989. Statistical Methods. 8th ed. Iowa State University, Ames.

29. Snijders, C. H. A. 1990. Fusarium head blight and mycotoxin contamination of wheat: A review. Netherlands J. Plant Pathol. 96:187-198.

30. Snijders, C. H. A., and Perkowski, J. 1990. Effects of head blight caused by Fusarium culmorum on toxin content and weight of wheat kernels. Phytopathology 80:566-570.

31. Sugiura, Y., Watanabe, Y., Tanaka, T., Yamamoto, S., and Ueno, Y. 1990. Occurrence of Gibberella zeae strains that produce both nivalenol and deoxynivalenol. Appl. Environ. Microbiol. 56:3047-3051.

32. Tanaka, T., Hasegawa, A., Matsuki, Y., Lee, U.-S., and Ueno, Y. 1985. Rapid and sensitive determination of zearalenone in cereals by highperformance liquid chromatography with fluorescence detection. J. Chromatogr. 328:271-278

33. Ueno, Y., and Ishii, K. 1985. Chemical and biological properties of trichothecenes from Fusarium sporotrichioides. Pages 307-316 in: Trichothecenes and Other Mycotoxins. J. Lacey, ed. John Wiley \& Sons, New York.

34. Wakulinski, W. 1989. Phytotoxicity of the secondary metabolites of fungi causing wheat head fusariosis (head blight). Acta Physiol. Plant. 11: 301-306.

35. Wang, Y. Z., and Miller, J. D. 1988. Effects of Fusarium graminearum metabolites on wheat tissue in relation to Fusarium head blight resistance. J. Phytopathol. 122:118-125.

36. Wong, L. S. L., Abramson, D., Tekauz, A., Leisle, D., and McKenzie, R. I. H. 1995. Pathogenicity and mycotoxin production of Fusarium species causing head blight in wheat cultivars varying in resistance. Can. J. Plant Sci. 75:261-267. 